$\xi=-1$

\title{
Performance evaluation of vanets using wireshark
}

\author{
Shakeel Ahmed ${ }^{1 *}$, N.V.K. Ramesh ${ }^{2}$ \\ ${ }^{1}$ Electronics and Communication Engineering, K L E F, Vijayawada, India \\ ${ }^{2}$ Electronics and Computer Science Engineering, K L E F, Vijayawada, India \\ *Email: shakeel_be@yahoo.com
}

\begin{abstract}
Vehicular Adhoc Networks (VANETs) have become a hot area of research and development since few years. It is actually a subclass of Mobile Adhoc Networks (MANETs) where each node is a vehicle and when the nodes come in the communication range of each other, they form a network. In VANETs, Vehicle to Vehicle(V2V) and Vehicle to Infrastructure(V2I) communication takes place where nodes themselves acts as servers and /or clients for sharing \& exchanging of information. VANETs find applications in road safety also have the potential to improve traffic efficiency and convenience by communicating with peer vehicles and roadside units. As the nodes move with high speed, the topology is dynamically varying and designing a routing protocol for such a dynamically varying network is a challenge. Also other challenges of VANETs are security, connectivity among vehicles, cross layer design issues, mobility \& validation. In this paper, a VANET scenario is simulated using Wireshark Simulator in IEEE 802.16e (WiMAX) and IEEE 802.11p (WAVE) environment and the performance evaluation parameters like packet loss ratio and throughput. The simulation results are performed by scaling the network size using both the environments i.e., WiMax and WAVE and the results are evaluated by comparing throughput and packet loss ratio. WiMAX outperforms WAVE in a VANET environment in a big network.
\end{abstract}

Keywords: Packet Loss Ratio; Throughput; WAVE; WiMAX

\section{Introduction}

With the advancement in technology, vehicle industry has incorporated wireless communication technology in the vehicles so that vehicles can communicate with other peer vehicles on the road for exchange of information about the traffic and safety warnings. VANETs [1], [2], [3] uses vehicles as mobile nodes and are actually a subclass of MANETs. These nodes (vehicles) are equipped with sensors and Global Positioning System (GPS) receivers, known as on-board units. These nodes communicate with the stationary road side infrastructure. Thus, communication in VANETs usually is among V2V and V2I. In order to support the communication among vehicles i.e., V2V \& V2I, a 75MHz frequency spectrum in $5.9 \mathrm{GHz}$ band is allocated by FCC for short range communications.

Communication among vehicles can take place in three different types of architectures: 1. In Ad hoc type of architecture also called as Infrastructure-less, the vehicles communicate with each other without using any infrastructure such as base station or access point, 2. In Infrastructure-based architecture, WiMAX/3G/4G/LTE technology is used for the vehicles to interact with fixed base stations like road side units using internet and 3 . The third type is the hybrid architecture which combines the functionality of the above two architectures to provide a wide variety of applications to both the driver and passengers.

In this paper the hybrid architecture of VANETs is used to explore its functionality by using Worldwide Interoperability for Micro- wave Access (WiMAX) and Wireless Access in Vehicular Environments (WAVE).

Figure 1 gives the architecture of VANET. It consists of vehicles and base stations known as road side units [4]. The mobile nodes (vehicles) are equipped with onboard units (OBU) and the Road side units (RSU) are situated at certain distance and in turn connected to internet to broadcast information to vehicles. Each of the nodes acts as a router and also as a repeater and routes the information to other vehicles within its range. Basically there are two types of communication networks: $1 . \mathrm{V} 2 \mathrm{~V}$ and $2 . \mathrm{V} 2 \mathrm{I}$ in order to establish the network and to exchange information.

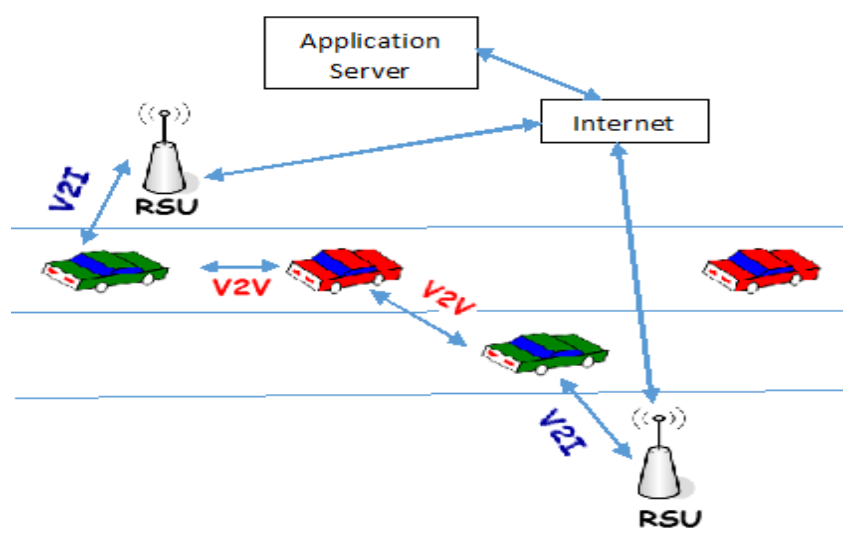

Fig. 1: VANET Architecture 
RSU can communicate with Internet and connect to the cloud to provide useful information.

This paper is proposed to create a VANET scenario using WiMAX and WAVE environment and evaluate its performance using Wireshark simulator. The rest of the paper is organized as follows. Section 2 gives WiMAX \& WAVE environment and the related work. Section 3 describes the simulator used. Section 4 presents the simulation setup using Wireshark simulator. Section 5 presents the simulation results and section 6I gives the conclusions.

\section{WiMAX, WAVE \& Related work}

\subsection{WiMAX Technology}

Worldwide Interoperability for Microwave Access (WiMAX) also known as IEEE 802.16e technology is an IP based, wireless broadband access technology [5] which performs similar to IEEE 802.11 network and have the coverage and guaranteed quality of service of infrastructure based cellular network. It is wireless communication system intended for metropolitan area network and is also known as IEEE 802.16. It provides high data rates of upto $70 \mathrm{Mbps}$ and coverage area upto 30 miles $(50 \mathrm{Kms})$ for fixed stations and 3-10 miles (5-15 Kms) for mobile stations. WiMAX operates on both licensed and unlicensed bands and the coverage area ranges about 4-5 miles including trees \& structures (i.e., NLOS environtment). In a line of site environment the range extends upto 10 miles (16 Kms).

\subsection{WAVE Technology}

WAVE (IEEE 802.11p) [6], is basically a version of IEEE 802.11 standard used for wireless communication among vehicles. Some of its features such as distributed medium access control mechanisms and simplicity are adapted from IEEE 802.11. This standard is used mostly for information exchange among vehicles, road side units, on board units and some fixed infrastructure like traffic signals. The standard supports both V2V and V2I communication at $5.9 \mathrm{GHz}$ band. A maximum of $27 \mathrm{Mbps}$ data rate and $100 \mathrm{Kmph}$ of mobility is supported by this standard.

\subsection{RELATED Work}

In [6], parameters like packet delay, packet loss ratio are evaluated and this QoS is calculated in both the WAVE and WiMax technologies implemented in VANET [7], [8], [9]. It is shown that if the length of the packet is small then WiMax is more suitable. Also, for long distance communication the packet loss ratio is less and $\mathrm{QoS}$ is more.

In [4], the authors concluded that WiMAX technology is preferable over WAVE or any other for VANETS in terms of performance compared to other wireless technologies. Thus, WiMAX is opted over WiFi, 3G or WAVE in this paper as it is more economical, have high download speed of upto $70 \mathrm{Mbps}$ and also says that a high QoS is ensured for both the video and voice applications.

\section{WIRESHARK Simulator}

The simulator used in this work is Wireshark. It is an open source network packet analyzer [10], [11], [12]. It is freely available and runs on both Windows and Unix platforms. It is capable of capturing the packets in a network and displays the data of a packet in detail. It is used as a measuring device to examine the inside of a network cable. It can be used by network engineers/administrators or developers to troubleshoot the various problems of networks, to analyze the security issues, to debug the protocols and to understand the inside of network protocols.
Wireshark first captures the data packets from the network then opens the files containing the data with tcpdump/ windump, wireshark and many other programs used for packet capturing.

It imports packets from text files, displays packets with detailed protocol information, saves packet data captured, export some or all packets, filter packets, tries to detect the packets, color the packet display using filters. In this paper Wireshark is used to create a scenario for analysis based on the assumptions made.

\section{Simulation}

\subsection{Simulation Environment \& Parameters}

The main aim of this simulation is to evaluate the performance of WAVE and WiMax technologies considering some urban scenarios. In this paper, Wireshark is used to create a scenario for analysis. Table I gives the platform for simulation.

\begin{tabular}{|l|l|}
\multicolumn{1}{|c|}{ Pable 1: Platform for Simulation } \\
\hline Bandwitdth & \multicolumn{1}{c|}{ Quantity } \\
\hline MAC Protocol & $1.76 \mathrm{GHz}$ \\
\hline Network Protocol & 802.11 \\
\hline Routing Protocol & Bellman Ford \\
\hline Modulation & BPSK \\
\hline TCP Window Size & $64 \mathrm{~KB}$ \\
\hline Network Path Latency & 60 milliseconds \\
\hline Number of Nodes & $\begin{array}{l}10,20,30,40, \\
50,60,70,80\end{array}$ \\
\hline
\end{tabular}

The simulation is carried out for a duration of 240 seconds by varying the size of network i.e., by increasing the number of nodes (vehicles) and calculating the parameters- packet loss ratio and throughput in vehicular environment. WiMax and WAVE technologies are used in the simulation.

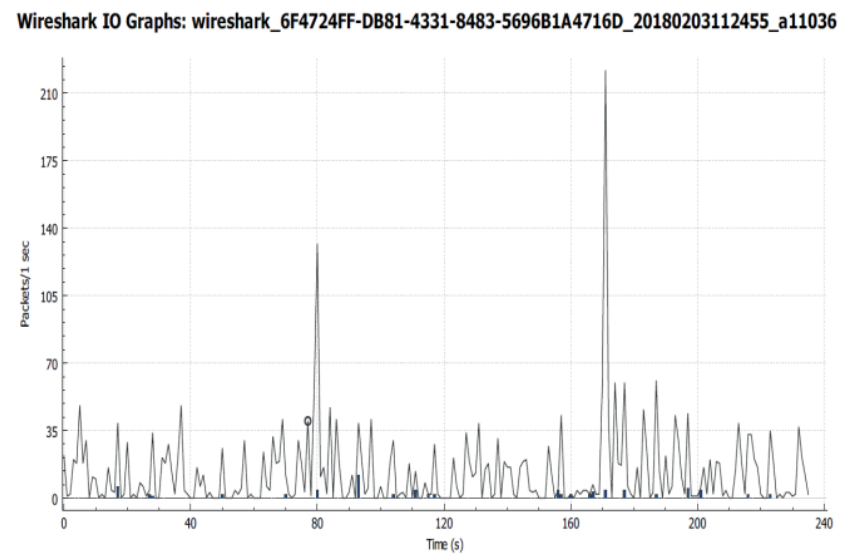

Fig. 2: Transmission of packets in Wireshark

Figure 2 shows transmission of packets by vehicles which are captured in Wireshark [14], [15].

Figure 3 gives the time at which each node starts transmission using TCP protocol. Each node here is assigned with an IP address. For example node with IP address 104.244.42.136 starts the communication with node of IP address 192.168.1.176. After the two way handshake i.e., after the transmission and reception of 
TCP request and the acknowledgement respectively the node forwards the data packet through other intermediate nodes. If the network is dense i.e., if the size of the network increases then the number of intermediate nodes (routers) will be more which degrades the performance of the network as the throughput of the network reduces due to long distance propagation. Also the amount of end-to-end delay increases.

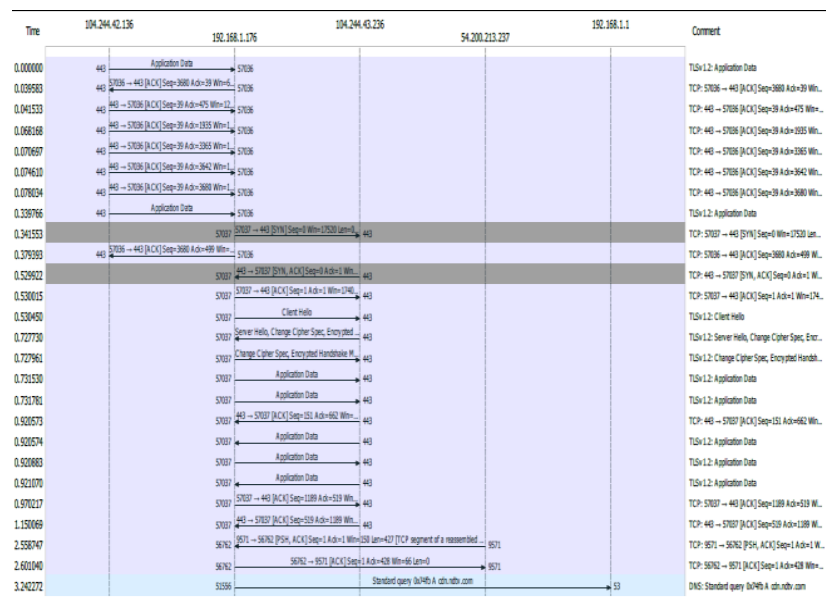

Fig. 3: Timing diagram for each node

\section{Simulation Results}

Throughput for every node and packet loss ratio are metrics of performance which need to be calculated.

Throughput is very important for data intensive VANET applications such as live images and videos. A fair enough throughput is essential for transfer of quality data. In this paper, average throughput per node is calculated as shown in figure 4. Here mean throughput is calculated as the ratio of size of the transmitted packet and packet delay. For example, figure 4 shows the throughput for a given node. $\mathrm{X}$-axis the simulation time and $\mathrm{Y}$ axis gives the average throughput for the node.

Throughput for 104.244.42.136:443 $\rightarrow$ 192.168.1.176:57036 (MA)

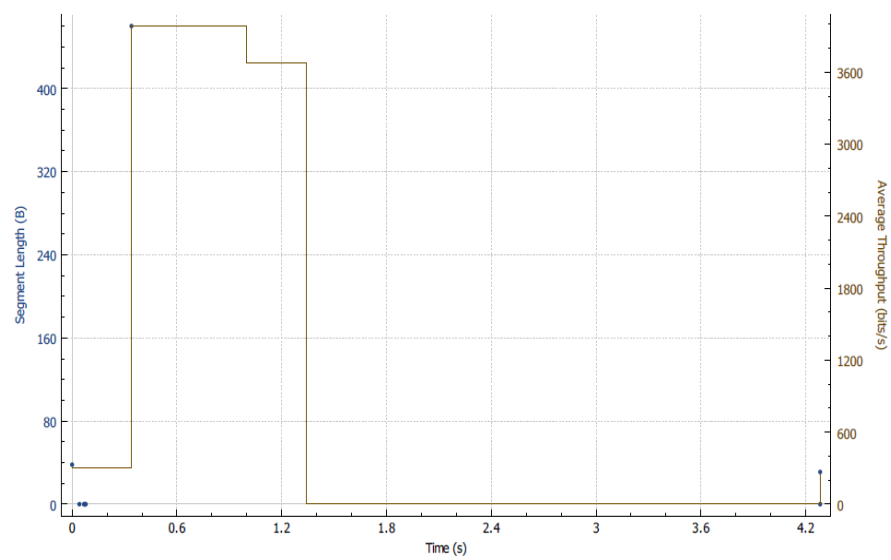

Fig. 4: Throughput for each node

Simulation is performed using both WiMAX \& WAVE technogies by varying the size of network. Initially, the network size is considered to be 10 with node 1 as source node and node 10 as destination node. Keeping the same source and destination nodes, the netowk size is increased to $20,30,40,50,60,70 \& 80$ and simulations are carried out for growing network sizes using WiMAX \& WAVE. The performance metric parameters, throughput and packet loss ratio is calculated for each of the simulation.

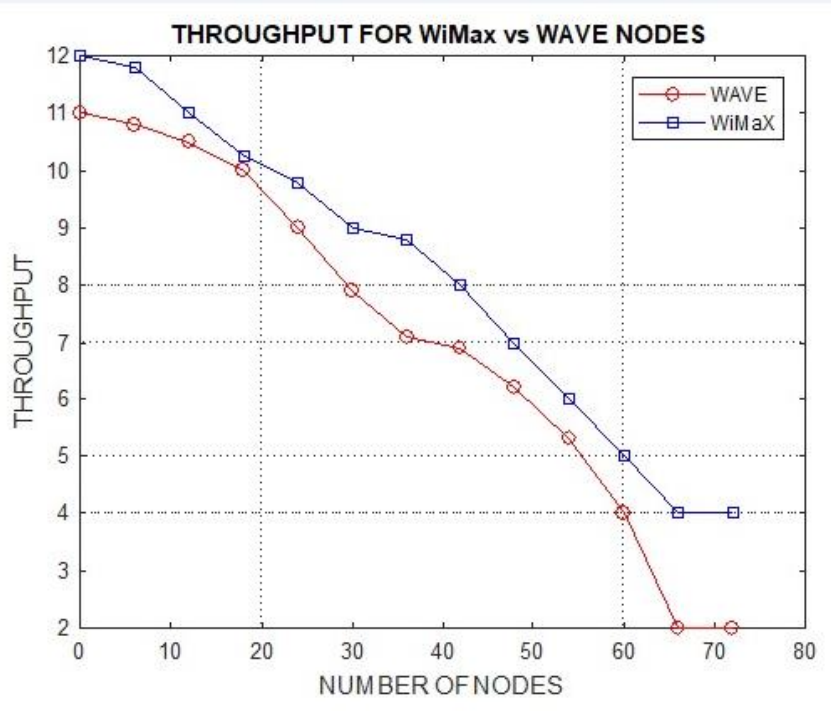

Fig. 5: Graph of Throughput for number of vehicles for WAVE and WiMAX

Figure 5 gives the graph of throughput for varying number of vehicles using WAVE \& WiMAX technologies. It is clear from the figure 5 that throughput is more for WiMAX technology in comparison with WAVE technology. Also throughput decreased as the size of the network increases.

Packet Loss Ratio is defined as ratio of packets loss due to collision and packets loss + packets received. This is required to calculate the efficiency of the network.

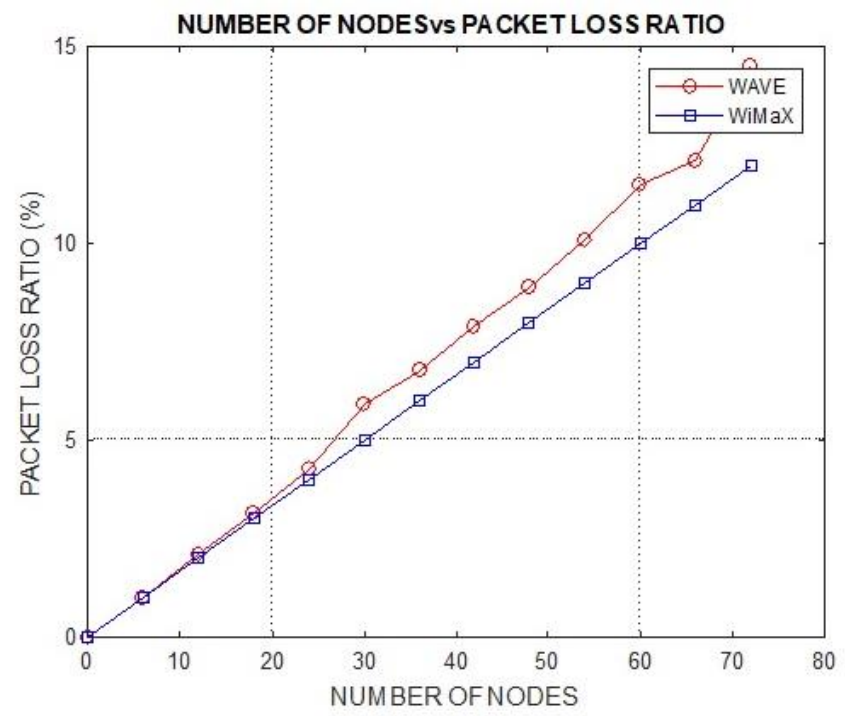

Fig. 6: Graph representing Packet Loss Ratio and number of nodes for WAVE and WiMAX

Figure 6 gives the graph of packet loss ratio with increasing network size. It is concluded from figure 6 that WiMAX outperforms WAVE even in case of packet loss ratio, also initially the packet loss is less for both the cases but gradually as the network size increases the packet loss ratio increases but compared to WAVE, WiMAX gives better performance.

\section{Conclusion}

The study of WAVE and WiMAX was done to find standard specifications. VANETs can be implemented using both the technologies. As the network grows then comes the choice of selecting the optimal technology. For which performance evaluation was done for WAVE and WiMAX environment by increasing the number of 
nodes (vehicles) in the given network. Two metrics: throughput and packet loss ratio are calculated for both the environments WiMAX and WAVE using Wireshark simulator. From simulation results it is concluded that WiMAX outperforms WAVE in a VANET environment in a big network.

\section{References}

[1] Nyoman Wira Prasetya; Tsang-Ling Sheu; Achmad Basuki; Muhammad Aziz Muslim, "A Pre-Emptive Traffic Model with Cluster-based TDMA for Vehicular Networks", IEEE International Conference on Software, Telecommunications and Computer Networks (SoftCOM), Sep. 2016, pp. 1-5.

[2] S. Zeadally, R. Hunt, Y. S. Chen, A. 1. and A. Hassan, "Vehicular ad hoc networks (V ANETS): status, results, and challenges",Springer Science and Business Media, LLC 2010, pp. $217-241$

[3] Wenshuang Liang, Zhuorong Li, Hongyang Zhang, Shenling Wang, and Rongfang Bie, "Vehicular Ad Hoc Networks: Architectures, Research Issues, Methodologies, Challenges, and Trends", International Journal of Distributed Sensor Networks, 2015.

[4] H. Hartenstein and K. P. Laberteaux, "A Tutorial Survey on Vehicular Ad Hoc Networks", IEEE Communications Magazine, June 2008, pp. 64-71.

[5] L. S. Mojela and M. J. Booysen, "On the use of WiMAX and Wi-Fi to provide in-vehicle connectivity and media distribution", Industrial Technology (1ClT), 2013 IEEE International Conference, pp. 1353-1358.

[6] R. Uzcategui and G. Acosta-Marum, "WAVE: a tutorial," Communications Magazine, IEEE, vol. 47, 2009, pp. 126-133.

[7] M. F. Iskander and Zhengqing Yu, "Propagation Prediction Models for Wireless Communication Systems", IEEE Transactions on Microwave Theory and Techniques, Vol. 50, No. 3, March 2002, pp. 662-673

[8] H. Hartenstein and K. P. Laberteaux, "A tutorial survey on vehicular ad hoc networks," IEEE Communications Magazine, vol. 46, no. 6, 2008, pp. 164-171.

[9] J. Harri, F. Filali, and C. Bonnet, "Mobility models for vehicular ad hoc networks: a survey and taxonomy," IEEE Communications Surveys and Tutorials, vol. 11, no. 4, 2009, pp. 19-41.

[10] Wireshark Foundation, "Wireshark - Go deep." Mar. 2010. [Online]. Available: http://www.wireshark.org/

[11] BzWorks PvtLtd, "PACKET SNIFFER - IEEE 802.15.4/ZigBeeTM ," 2006. [Online]. Available: http://www.bzworks.com/library/downloads/WiSens Datasheet.pdf

[12] S. J. Ban, H. Cho, C. Lee, and S. W. Kim, "Implementation of IEEE 802.15.4 Packet Analyzer," World academy of science, engineering and technology, vol. 35, pp. 66-69, Nov. 2007.

[13] Stephan Eichler, "Performance Evaluation of the IEEE 902.11p WAVE Communication Standard", WIVEC 2007.

[14] Di Guangqun, Hu Guijang. "Development and implementation of packet sniffer", CNKI: SUN: WJSJ.0.2009-21- 082.pp.1-5, 2009.

[15] Wireshark Documentation: http://www.wireshark.org/docs/wsug_html_chunked/index.html 